\title{
Heat recovery from biomass drying in energy systems
}

\section{Havlík, J. ${ }^{a^{*}}$; Dlouhý, T. ${ }^{\text {a }}$}

a Department of Energy Engineering, Faculty of Mechanical Engineering, Czech Technical University in Prague, Prague, Czech Republic.

*E-mail of the corresponding author: jan.havlik@fs.cvut.cz

\begin{abstract}
This paper deals with energy savings by the heat recovery of waste vapour from moist biomass drying in energy systems. Drying is an energy-intensive process. Energy consumption can be reduced by using indirect drying by recuperating the heat of waste vapour generated in the process; however the vapour is polluted by air and small mechanical particles. Experiments with green wood chips were realized on an indirect dryer with a condensing heat exchanger to experimentally verify the grade and conditions of heat recovery from waste vapour. On the basis of the experimental results, the potential of the heat recovery from waste vapour was evaluated.
\end{abstract}

Keywords: Indirect drying; Biomass; Waste vapour; Heat recovery. 


\section{Introduction}

Drying is an important process for creating optimal conditions for further material processing in many kind of industrial use. Industrial drying occurs by creating vaporization of the liquid by supplying heat to the wet feedstock. Heat may be supplied by convection (direct dryers) or conduction (contact or indirect dryers) [1]. In direct dryers, the material comes into direct contact with the heating medium, it is often hot air, flue gas or steam. In indirect dryers, the material and the heating medium (steam or hot water) are separated by a heat transfer surface. The heat is fed to the dried material through this surface, which defines the drying space. Generally, indirect dryers are more energy efficient. The typical energy consumption of direct dryers is in the range of $4.0-6.0 \mathrm{~kJ}$ per $\mathrm{kg}$ of evaporated water compared to indirect dryers which consume 2.8 - $3.6 \mathrm{~kJ}$ [1]. Additionally, if generated waste vapour is not mixed with drying or penetrating air, it leaves the dryer at a temperature close to $100{ }^{\circ} \mathrm{C}$ and its condensing heat can be recovered efficiently.

Drying is an energy-intensive process. The way to reduce the energy intensity of energy systems with drying is through the appropriate integration of contact drying with the use of heat recovery of the released waste vapour from the drying process [2]. The essence of heat recovery is vapour condensation together with gaining the vapour latent heat in an eligible heat exchanger. The main parameters limiting the further usage of this vapour are its purity and sufficient temperature level. The aim of the article is concentrated on an experimental evaluation of the potential and conditions of heat recovery from waste vapour which is generated during the drying of moist biomass in heating and power plants. Examples of heat recovery in the systems with integrated drying are shown in Fig. 1 [2]. The first case is a biomass fired steam power plant with an integrated indirect dryer heated
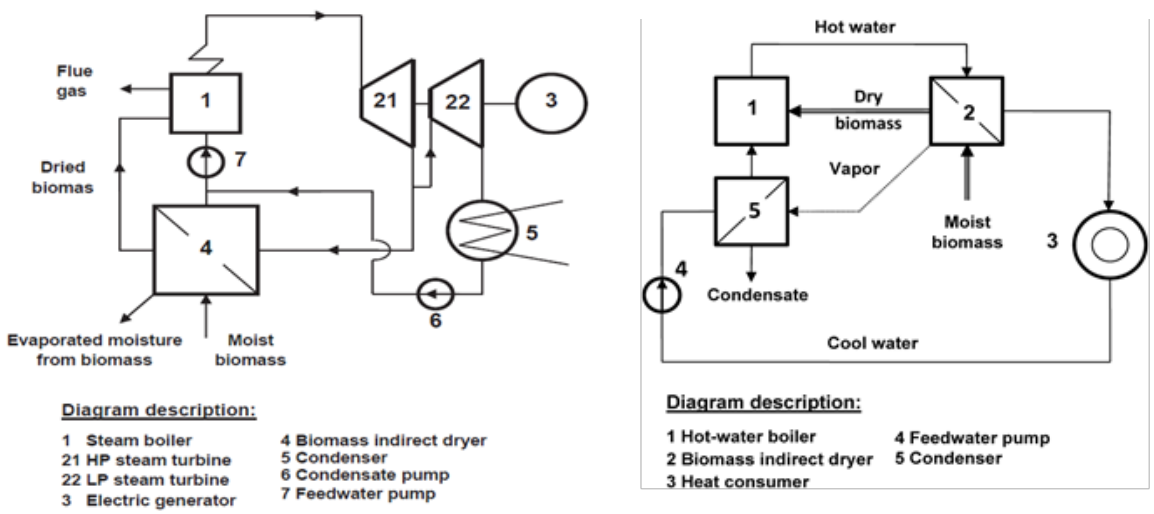

Fig. 1 Energy systems with indirect drying and waste vapour recuperation [2] 
by low-pressure steam extracted from the turbine. The integration of the dryer increases the cycle efficiency and power production and allows for further use of the waste vapour for preheating the feed water, external consumption in the heating system or preheating the air for pre-drying the biomass in the convective air dryer. The second system is the integration of an indirect dryer and a waste vapour condenser to a hot-water heating cycle. The indirect dryer is heated by hot water from the boiler while the cooled backwater is preheated in the condenser by the waste vapour from the dryer.

\section{Waste vapour recuperation}

Waste vapour from biomass drying may contain infiltrated air, aromatics or small mechanical particles released from the biomass during drying, which complicates its energy utilization. A big problem is the infiltrated air contained in the vapour which has a significant influence on the heat transfer during vapour condensation even in small concentrations. Specifically, this negative effect is caused by a reduction in the heat transfer coefficient and at the same time by a decrease in the condensation temperature during the process caused by the decreasing concentration of the condensing water vapour in the mixture [3].

If a mixture of steam with inert gases condenses, non-condensable gases cause a difference in the concentrations of the steam in the bulk gas mixture $C_{\infty}$ and at the condensation phase interface $C_{i}$ equally with corresponding steam partial pressures $p_{\infty}$ resp. $p_{i}$ and temperatures $T_{\infty}$ resp. $T_{i}$ (see Fig. 2 ). The molecules of steam diffuse through an inert gas towards the vapour-liquid interface and mass transfer occurs [3]. Steam concentrations also decrease along the length. On the other hand, in the case of pure steam condensation, the temperatures $T_{\infty}$ and $T_{i}$ are identical and constant during the process.

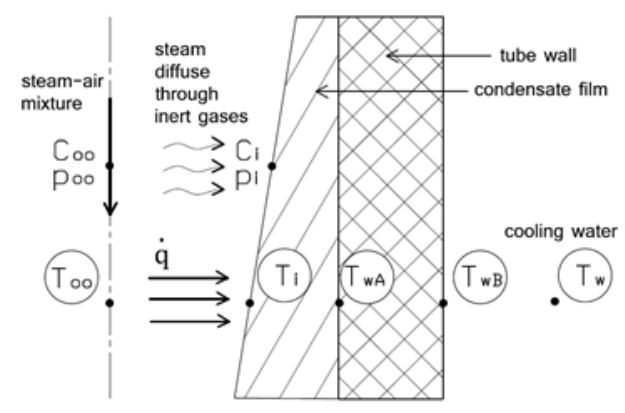

Fig. 2 Heat transfer in a condenser surface

In the examined case, a constant value of the heat flux $\dot{q}$ is considered for calculation of the heat balance (a constant heat flux from condensing steam, through condensate film, through the tube wall and to the cooling water). To determine the condensation temperature at the 
phase interface $T_{i}$, it is necessary to take into account the mass transfer generated by the different partial pressures at the interface between the gas and the liquid phases $p_{i}$, and at the bulk gas mixture $p_{\infty}$. The process of waste vapour condensation was studied inside the tubes of the vertical tube condenser, and the effect of the operation parameters and a comparison with the pure steam condensation process were analysed in detail in [4].

\section{Experimental set-up}

The experimental set-up consists of a steam heated indirect dryer and a recovery heat exchanger (Fig. 3).

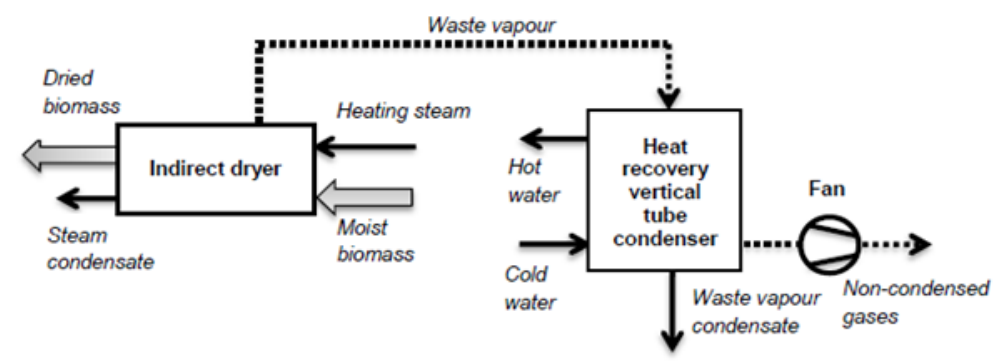

Fig. 3 Scheme of the experimental set-up

The dryer is designed as a rotating drum with a diameter of $600 \mathrm{~mm}$ and a length of $2000 \mathrm{~mm}$. The drum walls and wings for material blending consist of heated tubes with condensing steam inside. Dried material is continuously fed inside the drum by a screw conveyer, and leaves it on the opposite side. The drying capacity is about $20 \mathrm{~kg}$ of evaporated water per hour. The recovery heat exchanger is designed as a vertical shell-and-tube condenser. The condensing water vapour flows downwards inside vertical tubes and the cooling water flows in a counter current in the outer shell. This configuration is used due to the potential fouling of tubes by small solid particles in the waste vapour from the drying biomass. These particles, which can stick to the tube wall, are spontaneously carried away from the tubes by the condensate blowing out. The heating surface consists of the tube bundle with 49 tubes 854 $\mathrm{mm}$ in length and with an internal diameter of $24 \mathrm{~mm}$.

\section{Results}

Green wood chips with a moisture content from 62 to $66 \%$ were used for drying tests with waste vapour utilization (see Fig. 7). In the drying experiments, the moisture reduction in the drying material and the temperature of the outgoing waste vapour during the drying process was investigated. The entire drying process was described by a drying curve, which defines

$21^{\text {ST }}$ INTERNATIONAL DRYING SYMPOSIUM EDITORIAL UNIVERSITAT POLITÈCNICA DE VALÈNCIA 
the decrease in water content in the drying material over time [1]. An example of a drying curve for the dryer filled with material to $24 \%$ of the volume is shown in Fig. 4 . The initial drop in temperature of the waste vapour is influenced by the heating of the material from the exchange of the inlet temperature to the evaporated temperature and by the air content which is gradually pushed out from the dryer by the generated vapour.

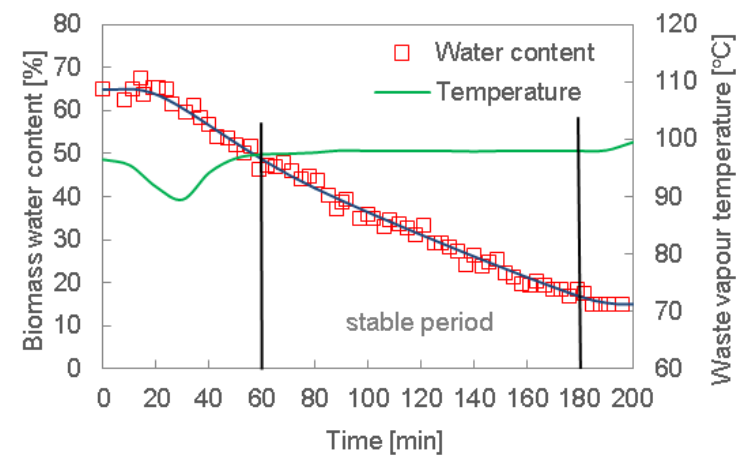

Fig. 4 Drying curve and corresponding waste vapour temperature

The stable temperature and waste vapour generating period between 60 and 180 min of the process in Fig. 4 was chosen for the evaluation of the waste vapour recovery potential. This state would correspond with the continuous operation of a large energy system. The investigated parameters were the amount of gained heat and its temperature level. In Table 1 , the measured parameters of the recovery heat exchanger operation and the evaluated results are shown for three repeated experiments. The cooling water flow rate was set to reach its temperature near to a constant level due to better evaluation of a decrease in the condensing vapour temperature along the tube length. The vapour velocity at the inlet of tubes was about $2 \mathrm{~m} / \mathrm{s}$.

Table 1. Utilization of waste vapour in the recovery heat exchanger

\begin{tabular}{lccc}
\hline \multicolumn{1}{c}{ Measurement } & $\mathbf{1}$ & $\mathbf{2}$ & $\mathbf{3}$ \\
\hline Waste vapour temperature - in / out $\left({ }^{\circ} \mathrm{C}\right)$ & $97.6 / 70.4$ & $97.3 / 70.2$ & $97.5 / 70.0$ \\
Air concentration in vapour - in / out $(\%)$ & $8.9 / 77.1$ & $10.1 / 77.4$ & $9,4 / 77.6$ \\
Water temperature - in / out $\left({ }^{\circ} \mathrm{C}\right)$ & $54.9 / 55.9$ & $54.7 / 55.7$ & $54.6 / 55.6$ \\
$\quad$ Results & & & \\
Waste vapour utilization - exp. (\%) & 97.1 & 96.7 & 97.0 \\
Waste vapour utilization - teor. (\%) & 98.8 & 98.5 & 98.3 \\
\hline
\end{tabular}

The temperature of the waste vapour leaving the actual biomass dryer was between 99 and $97{ }^{\circ} \mathrm{C}$ with an air content of about 8 to $10 \%$. The obtained results were compared with the 
theoretically balanced and calculated values based on principles for heat and mass transfer for the case of steam condensation in a binary mixture [3] according to the proposed and experimentally verified model of heat transfer for air-steam condensation in a vertical tube condenser described in [4]. The experimentally determined vapour recovery is in good agreement with the theoretically calculated values for this case. Experimental verification of this process makes it possible to estimate the operation of the device beyond the measured parameters and allows for future optimization.

The bulk gas temperatures $T_{\infty}$ and the condensation temperature $T_{i}$ are different and decreasing during condensation. For this reason, some of the waste vapour always flows through the exchanger and does not condense. The temperature courses along the length of the condenser tube for measurement no. 3 are shown in Fig. 5. These courses were theoretically calculated through the use of a model of the heat transfer in a vertical tube condenser [4] regarding the measured temperatures at the inlet and outlet. The growth of the cumulative heat output along the heat transfer area, respective the tube length, was also analysed in Fig 5. An important conclusion for the heat exchanger design and optimization is that the majority (approx. 80\%) of the recovered heat was obtained in the first half of the heat transfer surface. When increasing the amount of air to $20 \%$, the surface would be extended by more than $50 \%$ to maintain the heat exchanger output. On the contrary, decreasing the air content to $5 \%$ would result in a reduction to $72 \%$ of the surface.
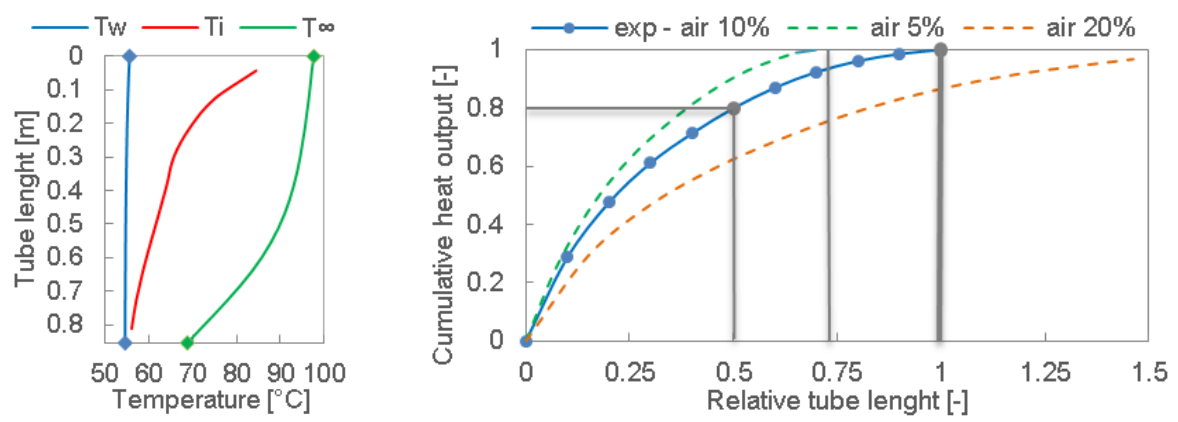

Fig. 5 Parameters change in waste vapour condensation process

In Fig. 6, the dependence of the utilization rate of waste vapour on the amount of infiltrated air (mass concentration) is shown for conditions approaching those of the actual industrial operation. The results are calculated according to the heat transfer model of the vertical tube condenser [4] for inlet temperatures of cooling water at $60{ }^{\circ} \mathrm{C}, 70{ }^{\circ} \mathrm{C}$ and $80{ }^{\circ} \mathrm{C}$. For an appropriate comparison of the heat recovery potential, the geometry of the heat exchanger is 
optimized for each case to heat the water by $5{ }^{\circ} \mathrm{C}$, to maintain a vapour velocity at the inlet of $2 \mathrm{~m} / \mathrm{s}$ and a temperature difference at the vapour outlet and water inlet of $2{ }^{\circ} \mathrm{C}$. This value was chosen as the limit, if we sought to achieve a lower temperature difference, it would be necessary to increase the size of the exchanger with very little impact on increasing the heat output.

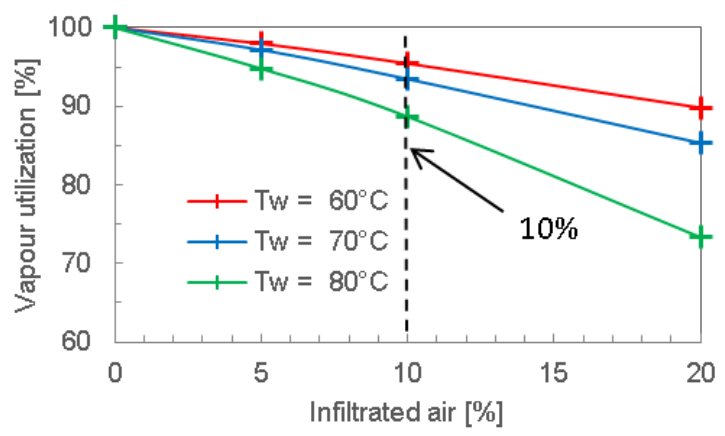

Fig. 6 Potential of waste vapour heat recovery and temperature level of this heat

For the value of infiltrated air of approximately $10 \%$ corresponding with the experiments above, the utilization rate would be above $90 \%$ for water heating at a temperature of $80{ }^{\circ} \mathrm{C}$, more for water temperatures of $70{ }^{\circ} \mathrm{C}$ and $60^{\circ} \mathrm{C}$. However, optimization of the device could result in lower amounts of infiltrated air and a higher level of vapour utilization. On the other hand, a higher level of air penetration significantly decreases the utilization rate.

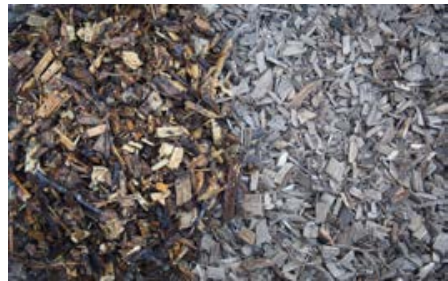

Fig. 7 Tested material - green wood chips before and after drying

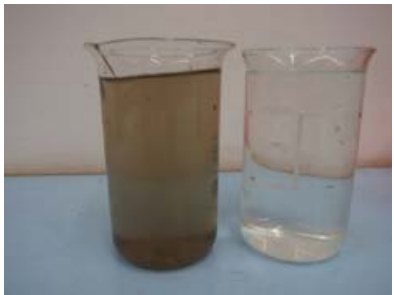

Fig. 8 Waste vapour condensate

The obtained condensate from biomass drying contains aromatic and dust particles which were released from the raw fuel during the drying process and carried away by the waste vapour from the dryer. For this reason, the condenser is designed with vertical tubes in which 
these particles are spontaneously washed out by the condensate. A comparison of the waste vapour condensate with the pure steam condensate is shown in Fig. 8.

After a series of experiments, a visual inspection of the heat exchanger surface was made and no visible fouling was observed. It can be stated that the proposed heat exchanger configuration is suitable for the condensation of waste vapour from biomass drying.

\section{Conclusion}

Based on the results of the experiments with the heat recovery from biomass drying, we can conclude that the waste vapour generated in indirect drying systems with limited air penetration has a sufficient potential for further energy utilization. The waste vapour exiting the tested drying device at atmospheric solution reached temperatures ranging from 97 to 99 ${ }^{\circ} \mathrm{C}$ with an air content of about $10 \%$. These conditions correspond with the potential of recovering more than $90 \%$ waste vapour condensing heat at the level of gained heat of $80{ }^{\circ} \mathrm{C}$ e.g. for water preheat.

The following principles are important in the design of a system with heat recovery of the waste vapour from indirect drying. Even small amounts of air in the waste steam cause a significant decrease in the heat transfer coefficient and the heat output of the recovery heat exchanger. The majority of the heat output is transferred in the first part of the recovery heat exchanger, which allows for economical optimization of its size. Waste vapour from some solid materials can contain small mechanical impurities, which can stick to the heating surface of the recovery heat exchanger, therefore it needs to be customized for cleaning. Condensers with vertical tubes seem to be a good solution in this case.

\section{Acknowledgement}

This work has been supported by the Technology Agency of the Czech Republic, project no. TJ01000192.

\section{References}

[1] Mujumdar, A. S. Handbook of Industrial Drying; Marcel Dekker: New York, 1990.

[2] Havlík J.; Dlouhý T. Integration of Biomass Indirect Dryers into Energy Systems. Journal of Chemical Engineering of Japan, 2017, 50 (10), 792-798.

[3] Hewitt, G. F.; Shires, G. L; Bott, T. R. Process Heat Transfer. Begell House: New York, 2000.

[4] Havlík, J.; Dlouhý, T. Condensation of the Air-steam Mixture in a Vertical Tube Condenser. EPJ Web of Conferences, 2016, 114, art. no. 02037. 